\title{
Primer Control System Cyber Security Framework and Technical Metrics
}

\author{
Wayne F. Boyer \\ Miles A. McQueen \\ May 2008
}

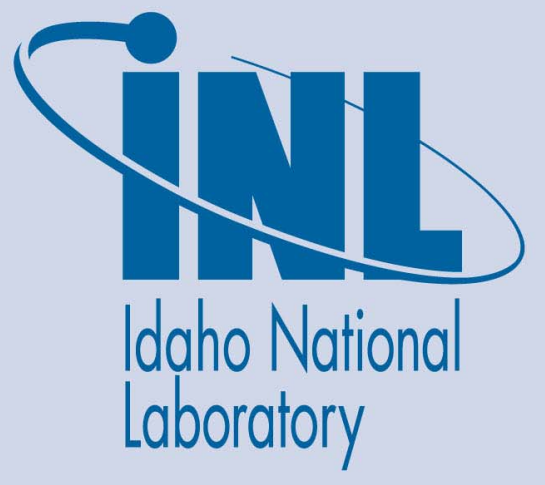

The INL is a U.S. Department of Energy National Laboratory operated by Battelle Energy Alliance 


\title{
Primer Control System Cyber Security Framework and Technical Metrics
}

\author{
Wayne F. Boyer \\ Miles A. McQueen
}

May 2008

US-CERT Control Systems Security Center

Idaho Falls, Idaho 83415 

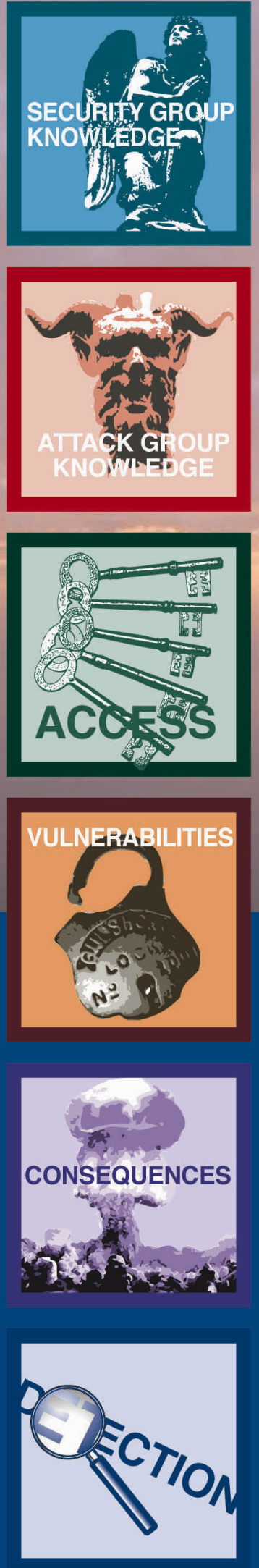

$\bar{E}$

\section{Primer}

Control System Cyber

Security Framework and

Technical Metrics

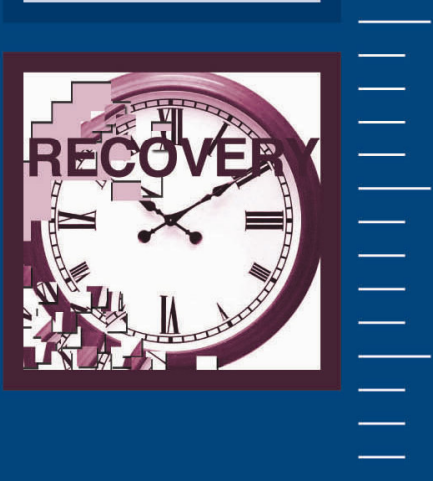

May 2008

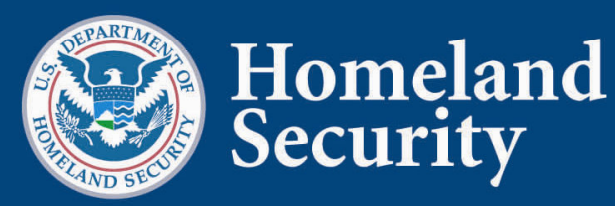





\section{EXECUTIVE SUMMARY}

The Department of Homeland Security National Cyber Security Division supported development of a control system cyber security framework and a set of technical metrics to aid owner-operators in tracking control systems security. The framework defines seven relevant cyber security dimensions and provides the foundation for thinking about control system security. Based on the developed security framework, a set of ten technical metrics are recommended that allow control systems owner-operators to track improvements or degradations in their individual control systems security posture.

This primer has been developed to assist you (owners and operators) in securing your control systems by applying the control system cyber security framework and implementing the ten technical security metrics. 


\section{CONTENTS}

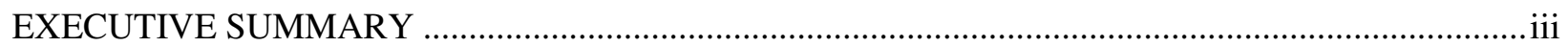

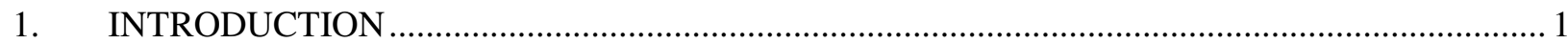

2. CONTROL SYSTEM CYBER SECURITY FRAMEWORK

$2.1 \quad$ Security Group Knowledge .......................................................................................... 3

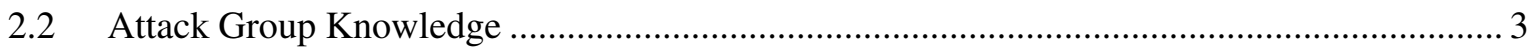

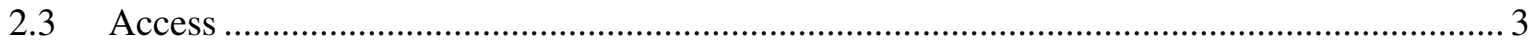

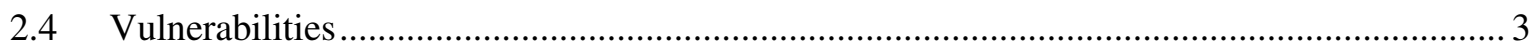

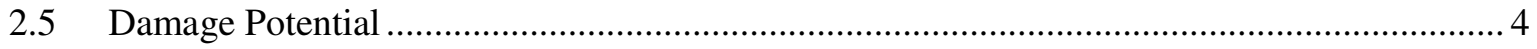

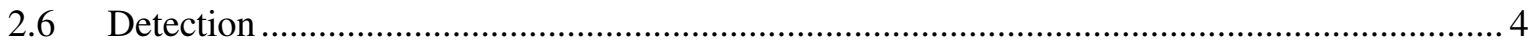

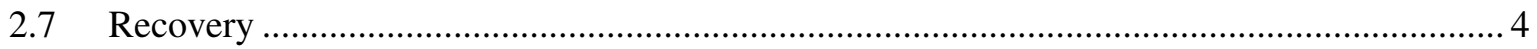

2.8 Security Dimensions Drive Security and Security Metrics.............................................. 4

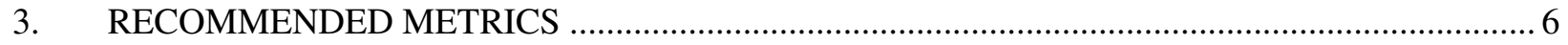

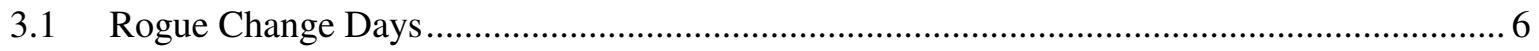

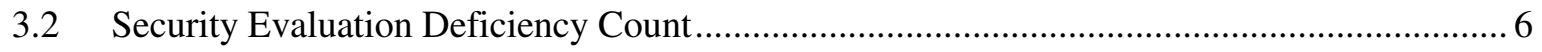

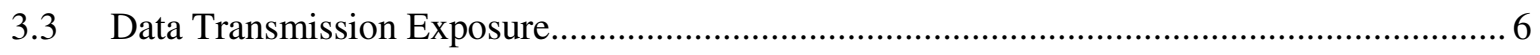

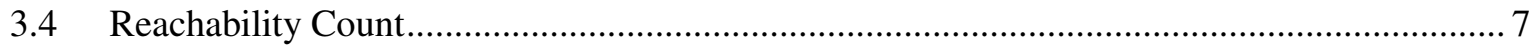

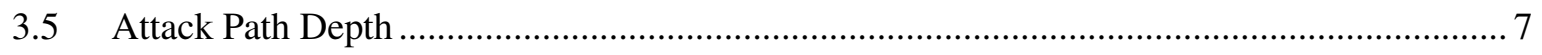

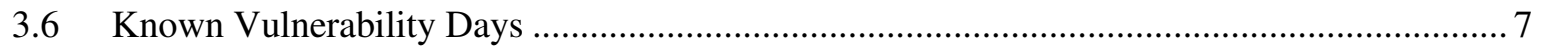

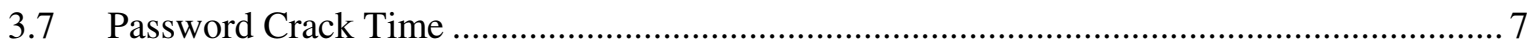

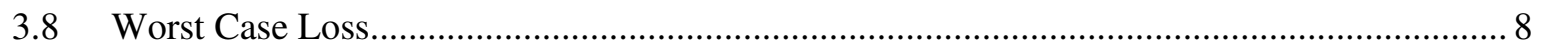

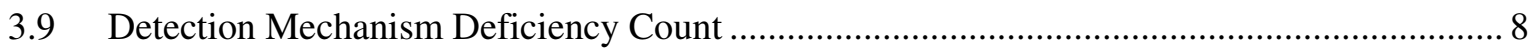

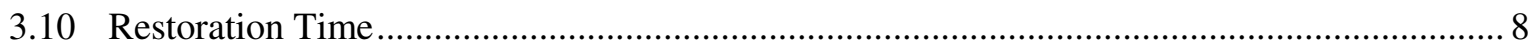

3.11 Specifications of Recommended Metrics................................................................... 8

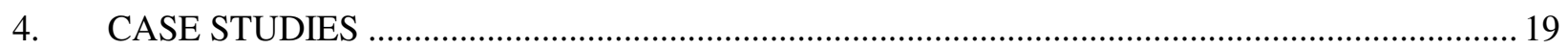

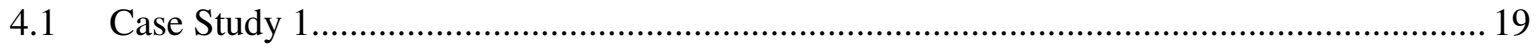

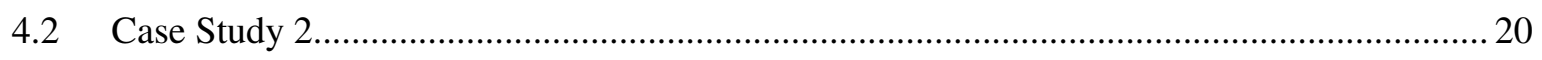

5. SURVEY OF PREVIOUSLY PROPOSED TECHNICAL METRICS ....................................... 22

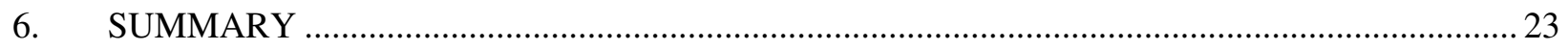

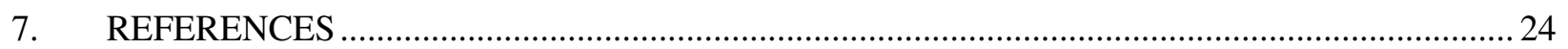

FIGURES

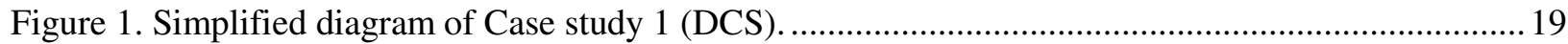

Figure 2. Simplified network diagram of Case Study 2 (SCADA) ........................................................ 20 
TABLES

Table 1. Seven Control System Cyber Security Dimensions...............................................................2

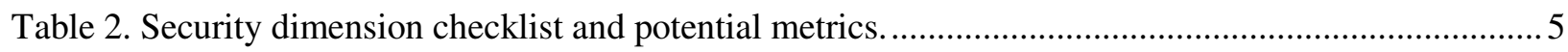

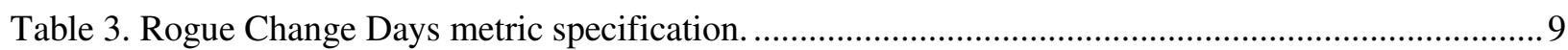

Table 4. Security Evaluation Deficiency Count metric specification. ...................................................... 10

Table 5. Data Transmission Exposure metric specification................................................................ 11

Table 6. Reachability Count metric specification............................................................................... 12

Table 7. Attack Path Depth metric specification. ................................................................................ 13

Table 8. Known Vulnerability Days metric specification.................................................................. 14

Table 9. Password Crack Time metric specification.......................................................................... 15

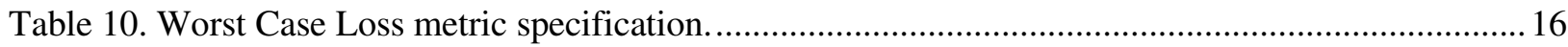

Table 11. Detection Mechanism Deficiency Count metric specification.................................................. 17

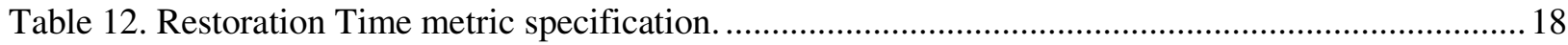

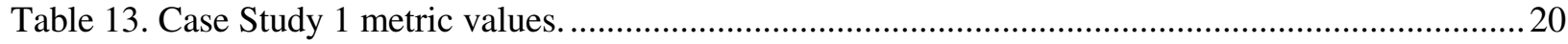

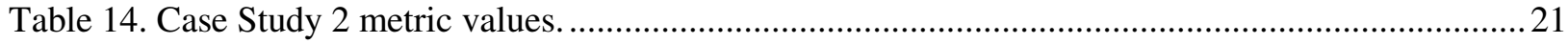




\section{INTRODUCTION}

Electronic control systems that operate much of the Nation's critical infrastructure are increasingly connected to public networks. Consequently, control systems and the associated critical infrastructure are at greater risk than before from externally initiated cyber attacks. To aid the mitigation of these increased risks, the Department of Homeland Security National Cyber Security Division funded development of (1) a control system focused cyber security framework to aid owner/operators in thinking about their control system's security posture and (2) a related set of meaningful technical metrics.

This primer describes the resulting framework and proposed metrics to you, the owner-operator, and is to be used as an aid when applying both the framework and metrics to your control systems. Rigorous application of the framework, when thinking about a control system's security posture, and consistent measurement and evaluation of the proposed metrics will support more effective security decisions for your systems.

The framework consists of seven security dimensions. Each of the security dimensions is related to risk. Unfortunately it is currently beyond the state-of-the-art and state-of-the-practice to quantify the relationship between any set of security dimensions and risk. However, based on case studies and review of control system security assessments, there is strong evidence that the framework's seven security dimensions capture many of the system attributes, which correlate with a control system's risk exposure. As a result, the seven security dimensions are the recommended foundation for thinking about the security of your system and are also the foundation for the ten recommended technical metrics. It is these ten technical security metrics that support you in assessing whether your security risk exposure is increasing or decreasing over time.

A metric is a standard of measurement. ${ }^{1}$ This primer presents ten useful and practical quantitative technical metrics. A cyber security technical metric is the security relevant output from an explicit mathematical model that makes use of objective measurements of a technical object. Other types of metrics (such as operational and organizational metrics, and metrics that are qualitative such as "low impact" or "highly unlikely") can provide insights about security but are beyond the scope of this primer.

The remainder of this primer consists of four additional sections. Section 2, "Control System Cyber Security Framework," discusses the framework of seven control system cyber security dimensions to aid you in thinking about the security of control systems; Section 3, "Recommended Metrics," presents and recommends one specific set of technical metrics that have been applied in case studies and demonstrated their value in improving control system security; Section 4, "Case Studies," discusses two case studies where the recommended technical metrics were applied to operational control systems; and Section 5, "Survey of Previously Proposed Technical Metrics," discusses a few previously proposed metrics, their strengths and weaknesses. 


\section{CONTROL SYSTEM CYBER SECURITY FRAMEWORK}

The control system cyber security framework consists of seven security dimensions and provides the foundation for the recommended metrics found later in this primer. Each of the seven dimensions of security represents an important aspect of the control system's security posture at a given point in time. An ideal value associated with each dimension indicates what would be needed in order to achieve security perfection along the associated dimension. The ideal values are provided to you as a target to move towards, not as a target that can be fully and completely met. The seven dimensions of security are defined in Table 1 and then discussed more fully in the rest of this section.
Seven Dimensions of Control System Cyber Security:

1. Security group knowledge

2. Attack group knowledge

3. Access

4. Vulnerabilities

5. Damage potential

6. Detection

7. Recovery

Table 1. Seven Control System Cyber Security Dimensions.

Security Dimension Definition

1. Security group Aspects of the system or associated management processes that impact the security group 's (SG) knowledge ability (the people who are directly responsible for the security of the control system) to

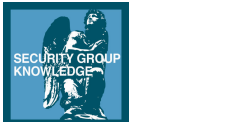

2. Attack group (AG) knowledge

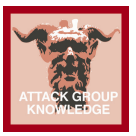

3. Access

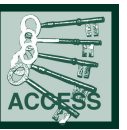

know the system and manage changes to the system. It includes (a) aspects of the system and processes associated with configuration management, (b) tools (or lack of tools) that support the tracking of changes, and (c) the collection and analysis of system logs and forensics.

Attributes of the system, processes, or actions that provide potential attackers with means to gain information about the system. It includes (a) software defects or configuration settings that return information when the system is probed by an unauthenticated user, (b) any information about the system that can be obtained from public sources, and (c) design or implementation weaknesses that allow a user with no authenticated privilege to gain information by listening on communication paths.

Attributes of the system design, configuration, or deployment that provide a potential attacker with the ability to send or receive data to/from a component of the control system from the attacker's location. This includes (a) physical access to control system components, (b) access to control system components through external and internal networks, and (c) access from internal components that may have been compromised. Access does not address whether or not the communication channel can be used to gain any useful information nor whether sending data can provide the attacker with any desired result.

4. Vulnerabilities Defects or weaknesses in the control system that can be exploited to gain unauthorized

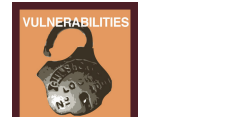
privilege. This excludes defects that allow information to be obtained once access is gained without also explicitly gaining privilege. If a single defect allows an attacker to gain information and also gain privilege, that defect is defined to be a vulnerability.

5. Damage potential The amount of loss that a malicious attacker has the power to cause once they have gained privilege on a control system. It does not include any weaknesses associated with the process of gaining malicious control. Although actual damage may be reduced by a quick response to an attack, this dimension does not include any effects associated with attack detection or control system recovery.

6. Detection

The ability to detect attacks and provide timely notification. This includes anti-virus software, IDS, IPS and system logging, whether detection mechanisms are in place and the effectiveness of those mechanisms.

7. Recovery

The ability to restore the control system from a compromised state to an uncompromised state. It includes the reliability of the backup and restore facilities and the time required to recover from an attack 


\subsection{Security Group Knowledge}

The first control system security dimension is Security Group (SG) knowledge. The security group represents those people in your organization who are directly responsible for the security of the control systems. Security risk is strongly correlated with the security group's knowledge of your system. In the ideal situation, the security group has perfect knowledge of the system including all the hardware and software components, network topology, communication paths, normal operational behavior, and even vulnerabilities. That knowledge is needed for your security group to effectively make security decisions that protect the control system from potential attackers. Any changes that occur to the control systems without the security group's knowledge may inadvertently introduce new vulnerabilities into the system and inhibit the introduction of reasonable mitigation measures. Perfect knowledge of the system implies a configuration management process that includes the security group in the planning of all changes and provides a mechanism for alerting the security group to any unauthorized changes.

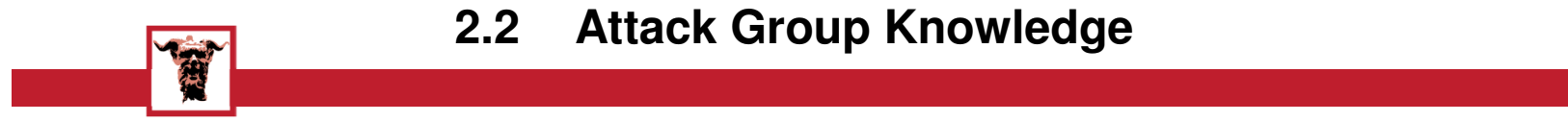

The second control system security dimension is Attack Group (AG) knowledge. The attack group represents any of the many potential adversaries in the world who might have interest in attacking your facility through cyber means. Security risk from targeted attacks is kept down when potential attackers are unable to obtain any information about your control system. Ideally, anyone who is not authorized to use the control system should be prevented from gaining knowledge of its design or configuration and be unable to obtain any information that would allow them to plan and execute an attack. This includes information an attacker might gain about the control system after they have compromised portions of it and information they might gain from other sources before the attack commences (e.g., a vendor's web site touting your facility as a success story).

Be aware that even approved users may become members of an attack group when their actions on the system go beyond what they are authorized to perform, whether inadvertently or intentionally (the "insider threat").

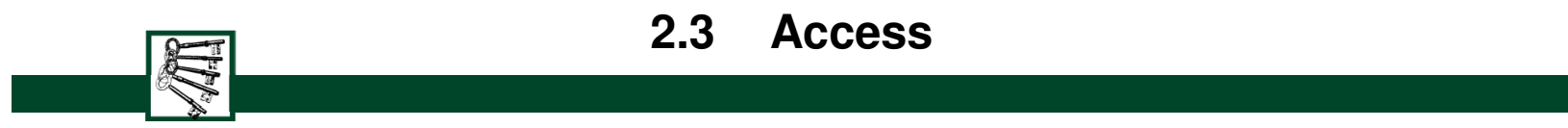

The third control system security dimension is Access. Even though authentication mechanisms are designed to prevent unauthorized use of data transfer paths, the existence of every path, authenticated or not, negatively impacts security risk. The ideal situation from a security perspective is to disallow any communication channels between the control system and any location where there are potential attackers. Although achievement of this ideal is not practical in most cases, the ideal includes the absence of any electronic connections between the Internet and the control system.

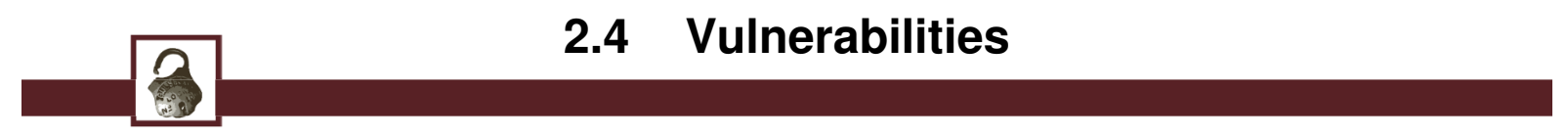

The fourth control system security dimension is Vulnerabilities. A vulnerability is any weakness or defect in the system that provides a potential attacker with a means to gain privilege intended for authorized users only. An exploit of a vulnerability leads to a compromise. An ideal system has no weaknesses and no defects. Unfortunately, all systems have weaknesses and if an attack group is targeting your facility they will be actively searching vulnerability disclosure sites and using techniques such as reverse engineering to find those weaknesses. 


\subsection{Damage Potential}

The fifth control system security dimension is Damage Potential. The ideal control system cannot cause damage even if the electronic networks are completely compromised by an attacker. Since risk is the expected value of loss, the damage potential is directly proportional to risk. The amount of damage that can be caused by a compromised control system is determined by the type of process that it controls and by the nature of engineered safety systems (e.g., physical safety mechanisms may be in place that prevents significant damage despite a successful attack on the electronic control system).

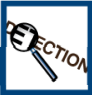

\subsection{Detection}

The sixth control system security dimension is Detection. An ideal control system includes detection mechanisms that alert the Security Group whenever there is an unauthorized event on the control system. Unauthorized events come in a wide variety of forms and would include activities such as an unauthorized user attempting to gain access to your control system or a counterfeit message from a front end processor to a remote terminal unit (RTU).

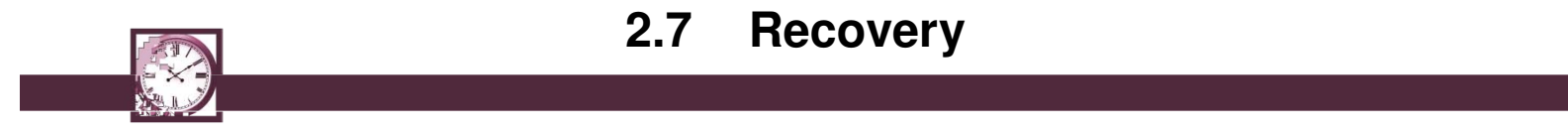

The seventh control system security dimension is Recovery. An ideal control system can be restored to an uncompromised state immediately after an attack is detected. Recovery time is related to Damage Potential because the cost of a successful attack correlates with the length of time that the control system is in a compromised state. Damage will tend to be less severe if the time to recover is minimized. However, the relationship between Recovery Time and Damage Potential is highly non-linear and highly system dependent.

\subsection{Security Dimensions Drive Security and Security Metrics}

Although perfection may not be feasible for any of these seven dimensions of control system cyber security, the measurement of how nearly the system approaches the ideal in each dimension is a useful way to think about the security risk to your system. Table 2 is a check list that can help you begin to consider each dimension, and ensure you are measuring and tracking some system attribute related to each cyber security dimension.

To support your efforts in establishing measures for each security dimension, ten technical security metrics have been defined with at least one of the metrics covering each security dimension. The ten metrics and their application are discussed in the next section. 
Table 2. Security dimension checklist and potential metrics.

\begin{tabular}{|c|c|c|}
\hline Security Dimension & System Attributes to Consider & Recommended metrics \\
\hline \multirow[t]{5}{*}{ 1. SG Knowledge } & Network diagrams up to date? & Rogue Change Days \\
\hline & Software change detection? & \multirow{4}{*}{$\begin{array}{l}\text { Security Evaluation Deficiency } \\
\text { Count }\end{array}$} \\
\hline & Hardware change management? & \\
\hline & Has the system been scanned for vulnerabilities? & \\
\hline & Are all communication channels identified? & \\
\hline \multirow[t]{4}{*}{ 2. A } & Encrypted traffic? & \multirow[t]{4}{*}{ Data Transmission Exposure } \\
\hline & $\begin{array}{l}\text { System specifications removed from control } \\
\text { system? }\end{array}$ & \\
\hline & Open source information? & \\
\hline & Does the system respond to network probes? & \\
\hline \multirow[t]{3}{*}{ 3. A } & Are all allowed network channels needed? & \multirow{3}{*}{$\begin{array}{l}\text { Reachability Count } \\
\text { Attack Path Depth }\end{array}$} \\
\hline & Physical barriers to computer access? & \\
\hline & Is authentication required? & \\
\hline \multirow[t]{2}{*}{ 4. Vuln } & Known vulnerabilities? & Known Vulnerability Days \\
\hline & Are authentication credentials strong? & Password Crack Time \\
\hline otential & Safety systems in place? & Worst Case Loss \\
\hline \multirow[t]{2}{*}{ 6. Detection } & Antivirus software? & \multirow{2}{*}{$\begin{array}{l}\text { Detection Mechanism Deficiency } \\
\text { Count }\end{array}$} \\
\hline & IDS? & \\
\hline \multirow[t]{2}{*}{ 7. Recovery } & Backups of all critical software? & \multirow[t]{2}{*}{ Restoration Time } \\
\hline & $\begin{array}{l}\text { Backups or documentation of all critical } \\
\text { hardware configurations? }\end{array}$ & \\
\hline
\end{tabular}




\section{RECOMMENDED METRICS}

Ten recommended technical metrics are listed in Table 2. Each metric is associated with one security dimension and there is at least one metric associated with each of the seven cyber security dimensions. Each defined metric is an answer to the question: "What can be objectively measured on the system that is a reasonable representation of how nearly the system approaches the ideal of its associated security dimension?" As this is a framework, the metrics you choose may be different but there should be at least one metric for each of the seven security dimensions. These ten recommended metrics have been successfully applied to two case studies described below and will be described in sufficient detail to provide you with a starting point for your own metrics plan. It is most important that you use these examples to consider how the metrics framework can be applied to your system in a consistent manner over time to track progress.
Recommended Technical Metrics:

1. Rogue Change Days

2. Security Evaluation Deficiency Count

3. Data Transmission Exposure

4. Reachability Count

5. Attack Path Depth

6. Known Vulnerability Days

7. Password Crack Time

8. Worst Case Loss

9. Detection Mechanism Deficiency Count

10. Restoration Time

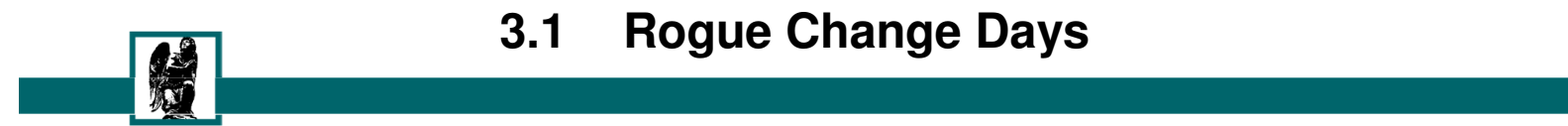

Rogue Change Days are the number of rogue changes multiplied by number of days the changes were unknown to the Security Group. A rogue change is any change to the system configuration without prior notification to the Security Group. For example, if two modems were added to the control system without the knowledge of the security group and this change was not discovered by the security group until 10 days later, this would add $2 * 10 \rightarrow 20$ rogue change days to the metric calculation. This is the first recommended metric for the security group knowledge security dimension. Its ideal value is zero.

\subsection{Security Evaluation Deficiency Count}

The Security Evaluation Deficiency Count is the number of control system network devices that have not undergone a security evaluation. This metric emphasizes the need to measure and track system knowledge about the security attributes of the control system. For example, if two RTUs that have not undergone security evaluations and one programmable logic controller (PLC) that has undergone security evaluation have been added to the control system, this would add a count of $3-1 \rightarrow 2$ to this metric calculation. This is the second recommended metric for the security group knowledge security dimension. Its ideal value is zero.

\subsection{Data Transmission Exposure}

Data Transmission Exposure is the unencrypted data transmission volume. A key assertion is that any data that can be monitored by a potential attacker increases the security risk. Some data are clearly more sensitive than others; however, to make the metric easier to obtain, it is simply a count of the number of clear text channels used by the control system. For example, if Telnet is used to connect to the engineering workstation of the control system from the Internet and if it is the only channel used for 
external access, then the value of the metric is 1 . Telnet channels are included in this metric because Telnet uses a clear text protocol that attackers can tap into to obtain passwords and other sensitive data. This is the recommended metric for the attack group knowledge security dimension. Its ideal value is zero.

\subsection{Reachability Count}

The Reachability Count is the number of access points in relation to a specific point of origin such as the Internet. A key assertion is that a reduction in the number of access points tends to reduce the cyber security risk. This metric is a count of the incoming and outgoing network communication channels plus the number of physical access data channels. For example, the reachability count (from the Internet) for a control system protected by a firewall is calculated as follows. Suppose the control system consists of ten machines with two open TCP/IP ports each, and suppose the firewall prevents access to one of the two ports on each machine but has no outgoing restrictions. The metric value is 10 incoming channels (one for each machine) plus 10 outgoing channels (one for each machine), $10+10 \rightarrow 20$. This is the first recommended metric for the access security dimension. Its ideal value is zero.

\subsection{Attack Path Depth}

The Attack Path Depth is the minimum number of independent, single-machine compromises required for a successful attack from an external network. This metric emphasizes the importance of having multiple layers of defense. You should avoid a protection configuration that can be defeated by a single exploit or compromise. A control system that has direct access through an external modem, for example, has a metric value of 1 because a successful attack requires only the compromise of a single device, even though it is connected to the Internet through a corporate IT network and through multiple firewalls. This is the second recommended metric for the access security dimension. Its ideal value is infinity.

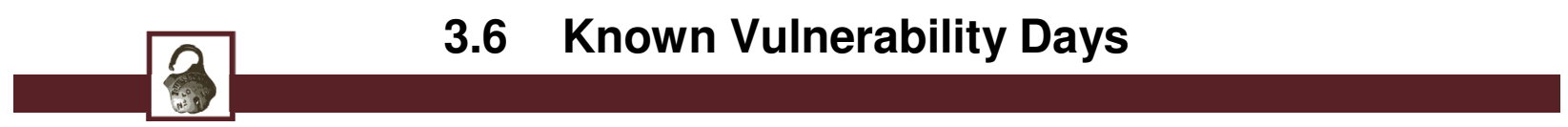

The Known Vulnerability Days metric is the sum of known and unpatched vulnerabilities, each multiplied by their exposure time interval. A key assertion is that the longer a vulnerability is known the greater the risk that it will be exploited. For example, if three vulnerabilities were publicly announced 2 weeks ago but still haven't been fixed on your system, it would add $3 * 14 \rightarrow 42$ known vulnerability days to this metric. This is the first recommended metric for the vulnerabilities security dimension. Its ideal value is zero.

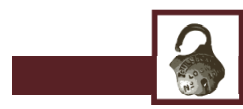

\subsection{Password Crack Time}

The Password Crack Time is the shortest time (in days) needed to crack a single password for any account on the system. This metric is a measure of the minimum amount of time an attacker would need to compromise the system by password cracking. For example, suppose the encrypted password files have been copied from all of the computers in the control room and the first of these passwords was cracked in 18 days and the second password was cracked in 30 days using John the Ripper. If no other passwords were cracked in fewer days the metric calculation would yield a value of minimum $(18,30) \rightarrow 18$ days. 
This is the second recommended metric for the vulnerabilities security dimension. Its ideal value is infinity.

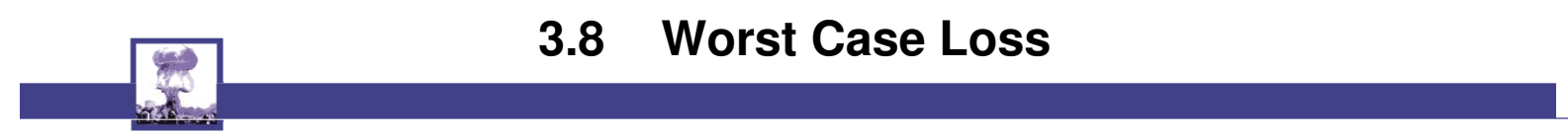

Worst Case Loss is the maximum dollar value of the damage/loss that could be inflicted by malicious personnel via a compromised control system. A key assertion is that system risk is strongly related to worst case loss. Although there can be successful attacks where the actual loss is much less than the worst case, a reduction in the worst case loss reduces the potential for loss and, therefore, reduces risk. For an example calculation of this metric consider a chemical plant in which a major explosion can be triggered by signals from the control system. The value of the metric is the estimated cost of the explosion in dollars including repairs, replacements and plant downtime. This is the recommended metric for the damage-potential security dimension. Its ideal value is zero.

\subsection{Detection Mechanism Deficiency Count}

The Detection Mechanism Deficiency Count is the number of externally accessible devices that do not have malware detection or attack detection mechanisms. A key assertion is that detection mechanisms reduce risk, especially when applied to devices that can be used as entry points for attacks. For an example calculation of this metric, suppose the control room has fifteen computers each with one or more currently enabled USB ports, and assume that twelve of the computers have antivirus installed but three do not. This would add $15-12 \rightarrow 3$ to this metric calculation, the recommended metric for the detection security dimension. Its ideal value is zero.

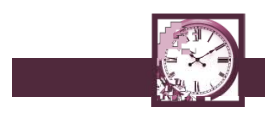

\subsection{Restoration Time}

The Restoration Time is the worst case elapsed time to restore the system to a known uncorrupted version. The metric can be determined by running a test to measure the actual time elapsed from a worstcase compromise to a fully restored and $100 \%$ operational system. If a test is not feasible and there have been no security events on your control system where the restoration time was tracked, you may have to resort to a pencil and paper exercise. For example, your exercise might assume a situation where all twenty computers in the control room have been compromised by a virus. Fortunately, the virus seems to be relatively benign so you are able to address one computer at a time. Perhaps, you decide to, one by one, take a computer off the network, operate in a degraded mode, clean the virus from that machine, and then reintroduce the computer to the network and get the applications in an up-to-date status. If this activity for a single machine takes 1-1/2 hours, it would yield a metric value of $20 * 90 \rightarrow 1,800$ minutes, the recommended metric for the recovery security dimension. Its ideal value is zero.

\subsection{Specifications of Recommended Metrics}

Detailed metric specifications for each of the ten recommended core metrics are listed in Tables 3 through 12. 
Table 3. Rogue Change Days metric specification.

\begin{tabular}{|c|c|}
\hline Metric Name & Rogue Change Days \\
\hline Brief Description & $\begin{array}{l}\text { The number of rogue changes multiplied by number of days the changes were unknown to } \\
\text { the Security Group (SG). A Rogue Change is any change to the control system } \\
\text { configuration without prior notification to the SG. }\end{array}$ \\
\hline Security Dimension & Security Group Knowledge \\
\hline Associated Principle & The system configuration should not be changed without the security group's knowledge. \\
\hline Key Assertion & $\begin{array}{l}\text { The security risk from changes to the system without notification to the security group is, } \\
\text { on average, worse than for changes that are announced in a well managed system. }\end{array}$ \\
\hline Units & RCD (Rogue Change Days) \\
\hline Range of Metric & Integer, 0 to infinity (best to worst) \\
\hline Strengths & A valid measure of the quantity of uncontrolled changes. \\
\hline Weaknesses & The metric does not include any measure of the actual security impact of changes. \\
\hline What Data is Collected & $\begin{array}{l}\text { The set of objects under change control must first be established and a version identifier } \\
\text { must be saved for each object to establish a baseline. Periodically the current version } \\
\text { identifier is scanned and compared to the previously saved identifier. Examples of objects } \\
\text { under configuration management are PLCs, HMIs, network devices attached to the local } \\
\text { network and critical computer files such as programs and graphical display pages. }\end{array}$ \\
\hline How Data is Collected & $\begin{array}{l}\text { One method for base lining computer files is to simply save a list of file names with } \\
\text { associated file dates. A rogue change is then detected when there is a difference between } \\
\text { the actual file date and the saved file date. Since file dates might be faked by an attacker a } \\
\text { more secure method is to apply a hash function to the content of each computer file to } \\
\text { calculate an identifier that can be saved with each file name and can be used to determine } \\
\text { when the file has changed. }\end{array}$ \\
\hline \multirow{6}{*}{$\begin{array}{l}\text { Mathematical Models } \\
\text { and/or Formulas }\end{array}$} & $\mathrm{S}_{\mathrm{T}}=$ An ordered set of version identifiers for all configured objects, measured at time $\mathrm{T}$. \\
\hline & $\begin{array}{l}\mathrm{S}_{\mathrm{T}+\mathrm{k}}=\text { An ordered set of version identifiers for all configured objects, measured at time } \\
\mathrm{T}+\mathrm{k} \text {. Note: Set } \mathrm{S}_{\mathrm{T}+\mathrm{k}} \text { may contain elements that are not in the set } \mathrm{S}_{\mathrm{T}} \text {, and set } \mathrm{S}_{\mathrm{T}} \text { may contain } \\
\text { elements that are not in the set } \mathrm{S}_{\mathrm{T}+\mathrm{k}} \text {. }\end{array}$ \\
\hline & $\mathrm{TSC}_{\mathrm{T}+\mathrm{k}}=$ Number of mismatches between sets $\mathrm{S}_{\mathrm{T}}$ and $\mathrm{S}_{\mathrm{T}+\mathrm{k}}$. \\
\hline & $\mathrm{CC}_{\mathrm{T}+\mathrm{k}}=$ Changes introduced into the system only after notification of the security group. \\
\hline & $\begin{array}{l}\mathrm{RC}_{\mathrm{T}+\mathrm{k}}=\mathrm{TSC}_{\mathrm{T}+\mathrm{k}}-\mathrm{CC}_{\mathrm{T}+\mathrm{k}} \text { is the number of rogue changes between the current measurement } \\
\text { of the system and the previous measurement of the system. }\end{array}$ \\
\hline & $\mathrm{RCD}=\mathrm{RC}_{\mathrm{T}+\mathrm{k}} * \mathrm{k}$ \\
\hline Tools & Tripwire $^{2}$ is an example of a tool that supports change auditing. \\
\hline $\begin{array}{l}\text { Data Collection } \\
\text { Frequency }\end{array}$ & $\begin{array}{l}\text { Because the data collection time interval is included in the calculation of the metric, there } \\
\text { is built-in incentive to measure often. }\end{array}$ \\
\hline Discussion & $\begin{array}{l}\text { RCD is somewhat conservative because the metric assumes the rogue change was } \\
\text { introduced immediately after the previous measurement. Only when one has a clear } \\
\text { understanding of all the devices, components, processes, and topology of a system is it } \\
\text { possible to achieve effective security management. This metric is practical for most } \\
\text { systems only after the deployment of tools that support strict change management. } \\
\text { Therefore, there should be an automated mechanism that allows the control system } \\
\text { manager to verify whether the current real system configuration matches the baseline. } \\
\text { Tools of this type are available or are feasible even though they have historically not been } \\
\text { widely used in control systems. }\end{array}$ \\
\hline
\end{tabular}


Table 4. Security Evaluation Deficiency Count metric specification.

\begin{tabular}{|c|c|}
\hline Metric Name & Security Evaluation Deficiency Count \\
\hline Brief Description & $\begin{array}{l}\text { The number of control system network devices that have not undergone a security } \\
\text { evaluation. }\end{array}$ \\
\hline Security Dimension & Security Group Knowledge \\
\hline Associated Principle & $\begin{array}{l}\text { The control system and its components should be evaluated and monitored for } \\
\text { vulnerabilities. }\end{array}$ \\
\hline Key Assertion & $\begin{array}{l}\text { Security evaluation of the network components identifies vulnerabilities and leads to } \\
\text { improved security of the control system. }\end{array}$ \\
\hline Units & Count. \\
\hline Range of Metric & Integer, 0 to infinity (best to worst). \\
\hline Strengths & $\begin{array}{l}\text { It is important to have some measure of how well the control system security has been } \\
\text { evaluated. This metric provides a simple measure. }\end{array}$ \\
\hline Weaknesses & The measurement requires technical judgment (not completely objective). \\
\hline What Data is Collected & $\begin{array}{l}\text { Identify all devices connected to the control system network. Identify which of those } \\
\text { devices have been evaluated from a security perspective. }\end{array}$ \\
\hline How Data is Collected & $\begin{array}{l}\text { First identify the system boundary, then identify all devices (machines) connected to the } \\
\text { network within that boundary. Count the devices within that boundary that have not } \\
\text { undergone a security evaluation. }\end{array}$ \\
\hline $\begin{array}{l}\text { Mathematical Models } \\
\text { and/or Formulas }\end{array}$ & Simple count. \\
\hline Tools & Control system diagram. \\
\hline & P\&IDs Piping and Instrument Diagrams. \\
\hline $\begin{array}{l}\text { Data Collection } \\
\text { Frequency }\end{array}$ & At least annually. \\
\hline Discussion & $\begin{array}{l}\text { Unresolved questions: Do security evaluations become obsolete with the passage of time. } \\
\text { If so, how much time makes them obsolete? When a network device is changed } \\
\text { significantly, the previous security evaluation is obsolete. How do you objectively } \\
\text { determine whether a given device has undergone a sufficient security evaluation? Even } \\
\text { though this metric has unresolved questions and requires some subjective judgment, it is } \\
\text { important enough to remain in the list of core metrics. }\end{array}$ \\
\hline
\end{tabular}


Table 5. Data Transmission Exposure metric specification.

\begin{tabular}{|c|c|}
\hline Metric Name & Data Transmission Exposure \\
\hline Brief Description & $\begin{array}{l}\text { Unencrypted data transmission volume. The number of unencrypted communication } \\
\text { channels in use by devices within the control system boundary. }\end{array}$ \\
\hline Security Dimension & Attack Group Knowledge \\
\hline Associated Principle & $\begin{array}{l}\text { The system should send no unencrypted information through networks that may be } \\
\text { monitored by potential attackers. }\end{array}$ \\
\hline Key Assertion & $\begin{array}{l}\text { As the quantity of clear text data transmission is reduced, the risk of disclosing sensitive } \\
\text { data to potential attackers is also reduced. }\end{array}$ \\
\hline Units & Count. \\
\hline Range of Metric & Integer, 0 to infinity (best to worst). \\
\hline Strengths & Can be objectively measured. \\
\hline Weaknesses & $\begin{array}{l}\text { Some data are clearly more sensitive than others, but, in order to be objective, this metric } \\
\text { only measures the potential for the interception of sensitive data rather than attempting to } \\
\text { measure the volume of sensitive data. The metric does not include any measure of data } \\
\text { previously made public that may be stored at an external location such as a search engine } \\
\text { cache. }\end{array}$ \\
\hline \multirow[t]{2}{*}{ What Data is Collected } & $\begin{array}{l}\text { Identify one or more network access points for data transmission monitoring. At least one } \\
\text { location should probably be outside the control system network perimeter. Points that may } \\
\text { be accessible to potential attackers are of the most interest. }\end{array}$ \\
\hline & $\begin{array}{l}\text { At each chosen access point, monitor data transmissions during a long enough time to } \\
\text { capture all the various types of traffic that occur during normal operations. Count the } \\
\text { number of unencrypted channels that terminate on a control system component. For a } \\
\text { TCP/IP network, count the number of IP-address, TCP-port pairs found in the network } \\
\text { traffic such that the TCP-port is associated with an unencrypted service and the IP-address } \\
\text { belongs to a component of the control system. File Transfer Protocol (FTP) and telnet are } \\
\text { examples of unencrypted services that would be included in this count. }\end{array}$ \\
\hline How Data is Collected & A network monitor is attached to each chosen network access point. \\
\hline $\begin{array}{l}\text { Mathematical Models } \\
\text { and/or Formulas. }\end{array}$ & $\begin{array}{l}\text { Simple count of observed unencrypted communication channels associated with control } \\
\text { system devices. }\end{array}$ \\
\hline Tools & Passive network monitor such as tcpdump. \\
\hline $\begin{array}{l}\text { Data Collection } \\
\text { Frequency }\end{array}$ & $\begin{array}{l}\text { Collect data whenever there is a configuration change that may affect network traffic. It } \\
\text { should be collected regularly (monthly, if possible), even if there are no known changes, to } \\
\text { verify that the same channels are still in use. }\end{array}$ \\
\hline Discussion & $\begin{array}{l}\text { Any data transmitted to external networks is potentially useful to an attacker. Ideally, there } \\
\text { should be no response to external network probes and no "clear text" transmissions. This is } \\
\text { a conservative metric because there is no attempt to determine the sensitivity of the data. } \\
\text { All data has the potential to provide useful information to an attacker; therefore, all data is } \\
\text { treated as equally sensitive. Some network paths are more critical than others, but during a } \\
\text { multistage attack, an attacker may gain access to an internal network by first penetrating } \\
\text { the system through an external network path. This metric does not include any measure of } \\
\text { the amount or nature of data that is made available to potential attackers in response to } \\
\text { probes, but vulnerability scanners often identify such behaviors. }\end{array}$ \\
\hline
\end{tabular}


Table 6. Reachability Count metric specification.

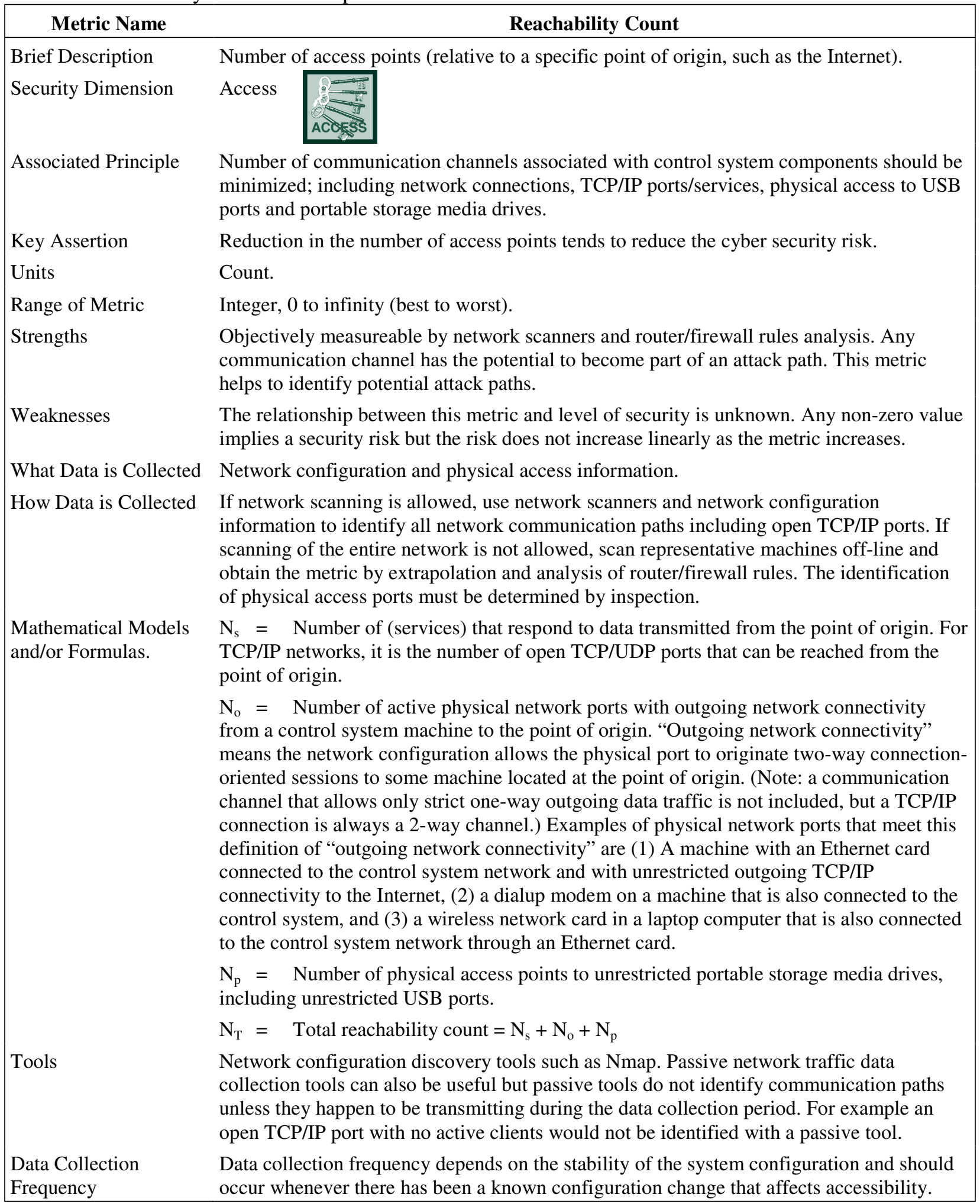


Table 6. (continued).

Metric Name

Discussion

\section{Reachability Count}

This metric applies to a specific point of origin. The system manager may choose to combine the network and physical reachability counts or track them separately.

The network point of origin of most concern is the Internet. Because of the possibility of penetration of the perimeter, the system manager may choose to also calculate this metric for points of origin within the network perimeter, such as at the DMZ or behind each firewall. The measurement of reachable ports/services includes all the cases of crafted packets that exploit known vulnerabilities in firewalls and routers, such as the spoofing of IP addresses and packet fragmentation to disguise the targeted TCP port number.

The point of origin for physical access may be outside of the perimeter or some other partially controlled area or combination of areas within the fence as defined by the system manager. Examples of restricted portable storage media drives that should not be included in the count of physical access points are:

USB ports that are disconnected, physically locked or blocked with glue

Host-based or device-based port encryption

Ports restricted by end-point control software.

Table 7. Attack Path Depth metric specification.

\begin{tabular}{|c|c|}
\hline Metric Name & Attack Path Depth \\
\hline Brief Description & $\begin{array}{l}\text { Minimum number of independent, single-machine compromises required for a successful } \\
\text { attack from an external network. }\end{array}$ \\
\hline Security Dimension & Acosśs \\
\hline Associated Principles & Compartmentalization, Defense in depth. \\
\hline Key Assertion & $\begin{array}{l}\text { Risk is reduced by increasing the number of independent steps required for a successful } \\
\text { attack. }\end{array}$ \\
\hline Units & Count. \\
\hline Range of Metric & Integer, 0 to infinity (worst to best). \\
\hline Strengths & This metric emphasizes the importance of having multiple layers of defense.. \\
\hline Weaknesses & $\begin{array}{l}\text { There may be common vulnerabilities on various paths of entry; therefore, the attack steps } \\
\text { may not be truly independent and this metric may be optimistic. }\end{array}$ \\
\hline What Data is Collected & $\begin{array}{l}\text { Identify all the network connections that form an unbroken set of links between the control } \\
\text { system and public networks. Find a path that traverses the fewest number of computers } \\
\text { between the public network and the control system. }\end{array}$ \\
\hline How Data is Collected & $\begin{array}{l}\text { If network scanning is allowed, use network scanners and network configuration } \\
\text { information to identify all network communication paths including open TCP/UDP ports. } \\
\text { If scanning of the entire network is not allowed, scan representative machines off-line and } \\
\text { obtain the metric by extrapolation and analysis of router/firewall rules. }\end{array}$ \\
\hline $\begin{array}{l}\text { Mathematical Models } \\
\text { and/or Formulas. }\end{array}$ & $\begin{array}{l}\text { Attack Path Depth = Minimum number of compromises required to reach any machine in } \\
\text { the set } S \text { from the public network by traversing reachable network paths. } S \text { is the set of } \\
\text { machines such that if any machine in the set } S \text { is compromised, then the attack is } \\
\text { considered to be successful. }\end{array}$ \\
\hline Tools & Network configuration discovery tools such as Nmap. \\
\hline $\begin{array}{l}\text { Data Collection } \\
\text { Frequency }\end{array}$ & $\begin{array}{l}\text { Data collection frequency depends on the stability of the system configuration and should } \\
\text { occur whenever there has been a known configuration change that affects accessibility. }\end{array}$ \\
\hline Discussion & This metric encourages network configurations that have several layers of protection. \\
\hline
\end{tabular}


Table 8. Known Vulnerability Days metric specification.

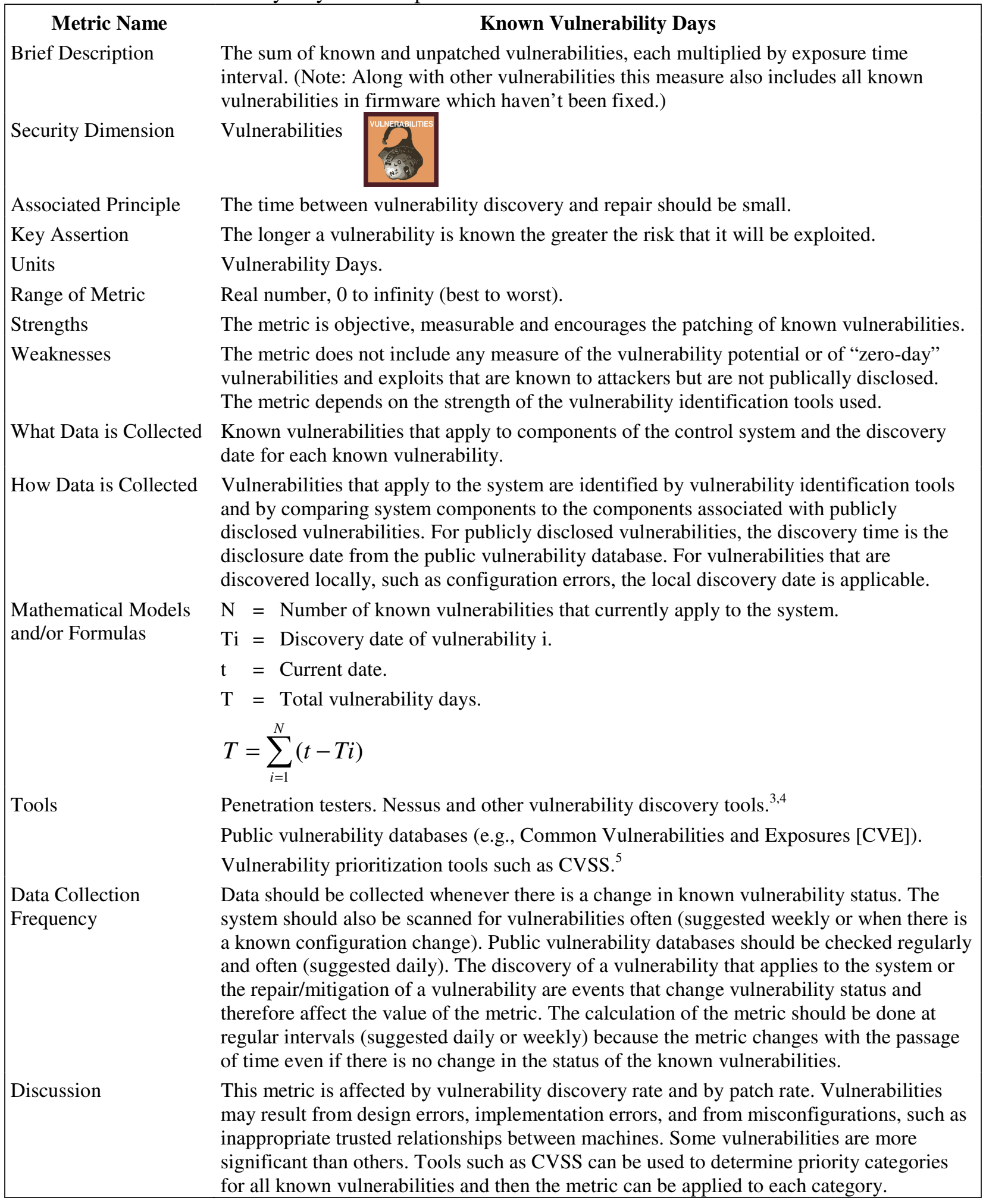


Table 9. Password Crack Time metric specification.

\begin{tabular}{|c|c|}
\hline Metric Name & Password Crack Time \\
\hline Brief Description & $\begin{array}{l}\text { The metric is the minimum time (in days) needed to crack a single password for any } \\
\text { account on the control system. }\end{array}$ \\
\hline Security Dimension & Vulnerabilities \\
\hline Associated Principle & Credential keys should be strong and should be changed regularly. \\
\hline Key Assertion & The control system security tends to improve when the password crack time increases. \\
\hline Units & Days. \\
\hline Range of Metric & Real number, 0 to infinity (best to worst). \\
\hline Strengths & $\begin{array}{l}\text { A valid measure of the time an attacker would need to compromise the system by } \\
\text { password cracking. Passwords are by far the most common form of authentication. }\end{array}$ \\
\hline Weaknesses & $\begin{array}{l}\text { Does not measure the strength of other authentication mechanisms. Does not account for } \\
\text { how well the hashed password data is protected. }\end{array}$ \\
\hline What Data is Collected & $\begin{array}{l}\text { Data collected for this metric is the encrypted password files (hashes) from all machines on } \\
\text { the system. For example, all password files from UNIX servers, configuration data for web } \\
\text { servers, database servers, Windows workstations, and control system Human Machine } \\
\text { Interface (HMI) machines. A password cracking tool is then applied to each password file } \\
\text { instance. }\end{array}$ \\
\hline How Data is Collected & $\begin{array}{l}\text { Copy account data for each instance of an operating system and each instance of an } \\
\text { application that implements authentication. For UNIX type machines the password hash } \\
\text { file or shadow file is simply copied. For windows machines a password hash file is created } \\
\text { from registry data by using the Pwdump }{ }^{6} \text { freeware. }\end{array}$ \\
\hline $\begin{array}{l}\text { Mathematical Models } \\
\text { and/or Formulas }\end{array}$ & Password Crack Time Metric $=$ Minimum time to crack any password on the system. \\
\hline Tools & $\begin{array}{l}\text { Password cracking tools are available commercially and for free download. Examples are } \\
\text { LOphtCrack } \\
\text { from and John the Ripper. }\end{array}$ \\
\hline $\begin{array}{l}\text { Data Collection } \\
\text { Frequency }\end{array}$ & $\begin{array}{l}\text { Data should be collected whenever passwords change. Password cracking software should } \\
\text { run continuously until there are new hash data or until all passwords are cracked. }\end{array}$ \\
\hline \multirow[t]{2}{*}{ Discussion } & $\begin{array}{l}\text { This metric is an important measure because passwords are by far the most common form } \\
\text { of authentication. The older a password becomes the more likely it is to be compromised, } \\
\text { therefore the system manager may choose to track password age as well as password crack } \\
\text { time. }\end{array}$ \\
\hline & $\begin{array}{l}\text { This metric is independent of password policies because it measures the least amount of } \\
\text { time an attacker would need to crack a password if the encrypted password data is } \\
\text { available to the attacker. If a very weak password is used (including a default vendor } \\
\text { supplied password), an attacker can guess the password without obtaining the encrypted } \\
\text { password files and this metric would detect that high risk situation because good password } \\
\text { cracking tools can crack very weak passwords virtually instantly. Passwords used for } \\
\text { authentication at the perimeter are particularly important and, therefore, perhaps crack time } \\
\text { for those passwords should be measured separately from other passwords used on the } \\
\text { system. The security group should ensure that vendor supplied passwords and passwords } \\
\text { commonly used by maintenance personnel are included in the password cracker's } \\
\text { dictionary. }\end{array}$ \\
\hline
\end{tabular}


Table 10. Worst Case Loss metric specification.

\begin{tabular}{|c|c|}
\hline Metric Name & Worst Case Loss \\
\hline Brief Description & $\begin{array}{l}\text { The maximum dollar value of the damage/loss that could be inflicted by malicious } \\
\text { personnel via a compromised control system. }\end{array}$ \\
\hline Security Dimension & Damage Potential \\
\hline Associated Principle & $\begin{array}{l}\text { Mechanisms that are independent of the control system should provide protection such that } \\
\text { the cost of damage due to control system malicious behavior is minimized. }\end{array}$ \\
\hline Key Assertion & Risk is strongly related to potential for loss. \\
\hline Units & Dollars. \\
\hline Range of Metric & Integer, 0 to infinity (best to worst). \\
\hline Strengths & This metric strongly correlates to risk. \\
\hline Weaknesses & $\begin{array}{l}\text { An objective measurement is usually not feasible. Estimation by careful analysis is } \\
\text { required. }\end{array}$ \\
\hline What Data is Collected & $\begin{array}{l}\text { Control system interfaces to the process under control. Detailed process and plant design } \\
\text { and configuration information. }\end{array}$ \\
\hline How Data is Collected & Facility documentation and expert elicitation. \\
\hline $\begin{array}{l}\text { Mathematical Models } \\
\text { and/or Formulas }\end{array}$ & Worst case system analysis. \\
\hline Tools & Standard safety analysis methods apply. \\
\hline $\begin{array}{l}\text { Data Collection } \\
\text { Frequency }\end{array}$ & $\begin{array}{l}\text { The metric should be reassessed whenever there is a system configuration change that may } \\
\text { impact the potential worst case loss. }\end{array}$ \\
\hline Discussion & $\begin{array}{l}\text { Although there can be successful attacks where the actual loss is much less than worst } \\
\text { case, a reduction in the worst case loss reduces the potential for loss and, therefore, reduces } \\
\text { risk. The worst case loss can probably be estimated from an existing safety analysis } \\
\text { associated with the plant. The metric is the answer to this question: "If the control system } \\
\text { is under the control of a malicious person, what damage can be done?" If safety systems } \\
\text { are not completely independent of the control system (for example a safety system } \\
\text { connected to the control system network) safety systems may also be compromised by an } \\
\text { attacker; therefore, it should not necessarily be assumed that such a safety system will } \\
\text { prevent damage when estimating the Worst Case Loss. }\end{array}$ \\
\hline
\end{tabular}


Table 11. Detection Mechanism Deficiency Count metric specification.

\begin{tabular}{|c|c|}
\hline Metric Name & Detection Mechanism Deficiency Count \\
\hline Brief Description & Number of externally accessible devices without any malware/attack detection mechanism. \\
\hline Security Dimension & Detection \\
\hline Associated Principle & $\begin{array}{l}\text { The system should be constantly monitored for malicious behavior and alarms should be } \\
\text { raised when detected. }\end{array}$ \\
\hline Key Assertion & $\begin{array}{l}\text { Detection mechanisms reduce risk especially when applied to devices that can be used as } \\
\text { entry points for attacks. }\end{array}$ \\
\hline Units & Count. \\
\hline Range of Metric & Integer, 0 to infinity (best to worst). \\
\hline Strengths & Easy to obtain. \\
\hline Weaknesses & $\begin{array}{l}\text { The effectiveness of detection mechanisms is not addressed by this metric. Correlation to } \\
\text { risk is unknown. }\end{array}$ \\
\hline What Data is Collected & $\begin{array}{l}\text { Identify which devices are accessible externally. Identify the location of each detection } \\
\text { mechanism (Antivirus Software, Intrusion Detection System (IDS), etc.) }\end{array}$ \\
\hline How Data is Collected & System configuration information. \\
\hline $\begin{array}{l}\text { Mathematical Models } \\
\text { and/or Formulas }\end{array}$ & Simple count. \\
\hline Tools & Not applicable. \\
\hline $\begin{array}{l}\text { Data Collection } \\
\text { Frequency }\end{array}$ & Whenever the configuration changes. \\
\hline Discussion & $\begin{array}{l}\text { Although detection mechanisms such as Antivirus Software and Intrusion Detection } \\
\text { Systems (IDS) cannot be expected to detect every attack, they do detect some attacks and, } \\
\text { therefore, should be employed at network entry points. This metric encourages the use of } \\
\text { these mechanisms. }\end{array}$ \\
\hline
\end{tabular}


Table 12. Restoration Time metric specification.

\begin{tabular}{|c|c|}
\hline Metric Name & Restoration Time \\
\hline Brief Description & Worst case elapsed time to restore the control system to a known uncorrupted version. \\
\hline Security Dimension & Recovery \\
\hline Associated Principle & The time needed to restore the system to an uncorrupted state should be minimal. \\
\hline Key Assertion & $\begin{array}{l}\text { Risk is correlated with the amount of time needed to restore the control system to an } \\
\text { uncompromised state. }\end{array}$ \\
\hline Units & Minutes. \\
\hline Range of Metric & Real number, 0 to infinity (best to worst). \\
\hline Strengths & Simple model. \\
\hline Weaknesses & $\begin{array}{l}\text { On some systems it may be difficult to measure accurately. Many systems require } \\
\text { continual operation and there may be no known incidents that included full system } \\
\text { restoration. }\end{array}$ \\
\hline What Data is Collected & $\begin{array}{l}\text { Measure the actual time elapsed from "start" to a fully restored and } 100 \% \text { operational } \\
\text { system. }\end{array}$ \\
\hline How Data is Collected & $\begin{array}{l}\text { Record elapsed time during the recovery process. This data should be collected for actual } \\
\text { security events if they have occurred. If there have been no recorded security incidents a } \\
\text { test of the recovery process would include restoration of all software. }\end{array}$ \\
\hline \multirow{2}{*}{$\begin{array}{l}\text { Mathematical Models } \\
\text { and/or Formulas }\end{array}$} & $\mathrm{T}_{0}=$ Start time (Time compromise is detected, or test start time) \\
\hline & $\mathrm{T}_{\mathrm{r}}=$ Time at which recovery is complete and the system is $100 \%$ operational. \\
\hline Tools & $\begin{array}{l}\text { Restoration time }=\text { Maximum value of all instances of }\left(\mathrm{T}_{\mathrm{r}}-\mathrm{T}_{0}\right) \text {. } \\
\text { clock }\end{array}$ \\
\hline $\begin{array}{l}\text { Data Collection } \\
\text { Frequency }\end{array}$ & As needed. \\
\hline Discussion & $\begin{array}{l}\text { The metric can be determined by running a test to measure the actual time elapsed from the } \\
\text { "start" of worst case compromise to a fully restored and } 100 \% \text { operational system. If it is } \\
\text { impractical to perform that kind of test on an operational system, then this data should be } \\
\text { collected for actual security events if they have occurred. If a recovery test is not feasible, } \\
\text { then a worst case recovery analysis may be used to estimate recovery time. }\end{array}$ \\
\hline
\end{tabular}




\section{CASE STUDIES}

The recommended technical metrics were applied in two case studies of operating control systems. These studies helped to identify the practical limitations associated with data collection and provide specific examples of how the metrics could be obtained and used. The Control System Technical Security Metrics Report ${ }^{9}$ gives a detailed account of these case studies.

\subsection{Case Study 1}

Case Studies of Operating Control Systems:

1. Distributed control system for a chemical processing plant

2. SCADA System used for power distribution

The first case study was of a Distributed Control System (DCS) for a chemical processing plant. Figure 1 is a simplified network diagram of the case study system.

Notice that the system is connected to the Internet through the corporate network. The router that provides connectivity between the corporate network and the local TCP/IP network restricts access to the control system with an access control list so that only the incoming TCP/IP connections with origination addresses that match the control list are allowed through the router. The system boundary is defined to be the processing plant and the control system networks that are within the control room. A dedicated telephone line connects the control system to the plant data archive system, which has direct connectivity to the corporate network. The corporate network affects the security of the control system, but, for this study, the corporate network was not considered to be part of the system.

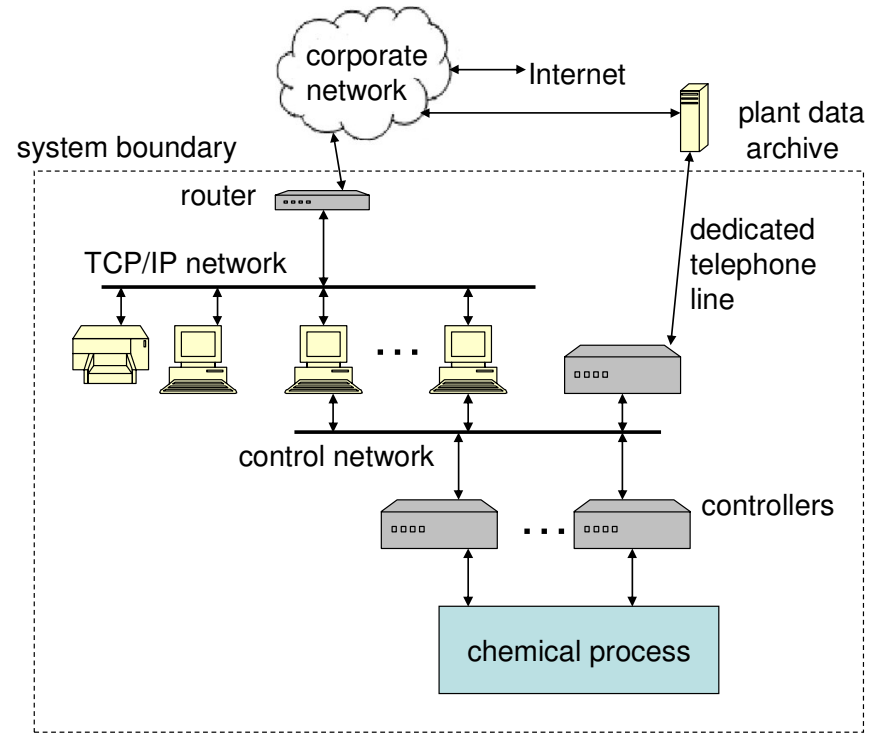

Figure 1. Simplified diagram of Case study 1 (DCS).

The DCS for this case study consists of a TCP/IP network that provides connectivity for eleven workstations and two printers, and a proprietary control network that provides connectivity to approximately thirty distributed controller nodes that control and monitor the plant. The workstations on the TCP/IP network consist of standard IT hardware, standard IT operating system software, and application software supplied by the DCS vendor. The controller nodes consist of specialized control hardware and software supplied by the same DCS vendor.

The metric values obtained for Study 1 are shown in Table 13, which also shows the ideal value and suggested target value for each metric. The suggested target value is the value we estimate could be obtained by changing the system configuration to improve security while retaining required functionality. This case study verified that the values of the metrics listed in Table 13 could be obtained using tools that are readily available. 
Table 13. Case Study 1 metric values.

\begin{tabular}{|llll|}
\hline \multicolumn{1}{|c}{ Metric Name } & \multicolumn{1}{c}{ Metric Value } & Ideal Metric Value & \multicolumn{1}{c|}{$\begin{array}{c}\text { Suggested Target } \\
\text { Value }\end{array}$} \\
\hline Rogue Change Days & 0 & 0 & 0 \\
Password Crack Time & $>30$ days & $\infty$ & $>30$ days \\
Data Transmission Exposure & 23 & 0 & 1 \\
Reachability Count $\left(\mathrm{N}_{\mathrm{T}}\right)^{\mathrm{a}}$ & 164 & 0 & 1 \\
- Physical $\left(\mathrm{N}_{\mathrm{p}}\right)$ & 2 & 0 & 0 \\
- Services $\left(\mathrm{N}_{\mathrm{S}}\right)$ & 149 & 0 & 1 \\
- Outgoing $\left(\mathrm{N}_{\mathrm{o}}\right)$ & 13 & 0 & 0 \\
Attack Path Depth & 2 & $\infty$ & 4 \\
Worst Case Loss & $\$ 100 \mathrm{M}$ & $\$ 0$ & unknown \\
Detection Mechanism Deficiency Count & 12 & 0 & 0 \\
Known Vulnerability Days (high priority) & 16,416 vuln. days & 0 & 0 \\
Known Vulnerability Days (low priority) & 15,877 vuln. days & 0 & 0 \\
Restoration Time & 120 minutes & 0 & 120 minutes \\
a. $\mathrm{N}_{\mathrm{T}}=\mathrm{N}_{\mathrm{p}}+\mathrm{N}_{\mathrm{S}}+\mathrm{N}_{\mathrm{o}}$ & & & \\
\hline
\end{tabular}

\subsection{Case Study 2}

Case Study 2 was for a power distribution Supervisory Control and Data Acquisition (SCADA) system. Figure 2 is a simplified network diagram of the SCADA system showing the main components and connectivity. The SCADA controls seven power distribution substations. There is one PLC and one Voice over Internet Protocol (VoIP) phone physically located at each of the seven substations, twentyfive electric power meters connected directly to the network. One engineering workstation (EWS), two front-end processors (FEP), three human machine interface (HMI) hosts, one printer, and the SCADA firewall are all physically located in the main substation control room. All devices are logically connected to a single TCP/IP network through routers and switches. The only connection from the SCADA network to the external network is through the SCADA firewall with a Virtual Private Network

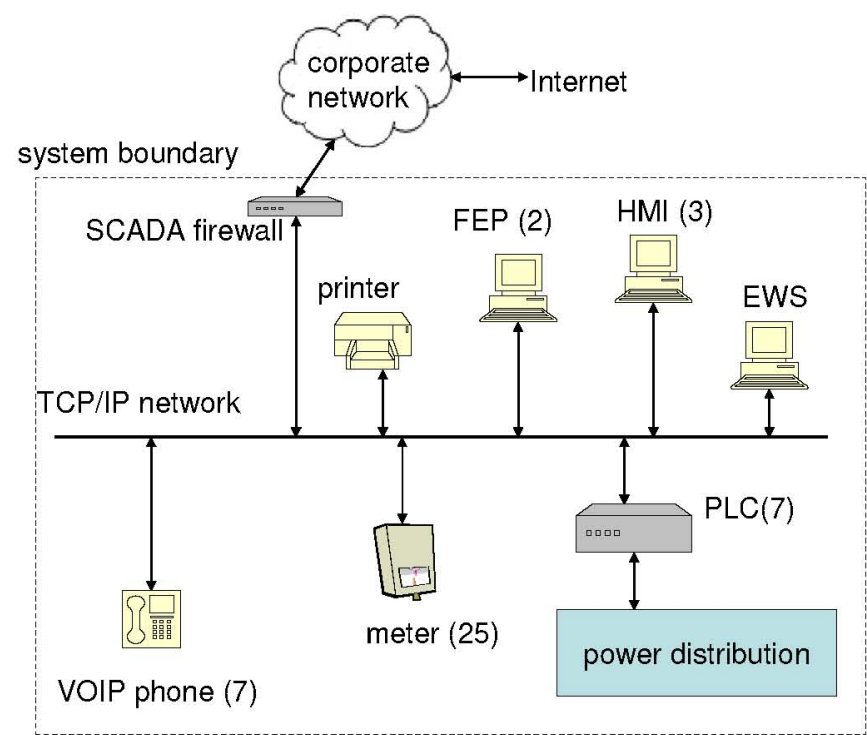

Figure 2. Simplified network diagram of Case Study 2 (SCADA). (VPN).

Data were collected by testing on a duplicate laboratory system when feasible, rather than the live SCADA system, to avoid potential disruption of operations. For the cases where the laboratory system did not provide sufficient information, data was also collected on the live operating SCADA system. 
All of the passwords for this case study,were cracked in less than one day using John the Ripper. The password hashes were LAN Manager hashes that are known to be easy to crack. The metric values obtained for Case Study 2 are shown in Table 14.

Table 14 also gives the ideal value for each metric and the suggested target value. The suggested target value is the value we estimate could be obtained by changing the system configuration as suggested by the comments in the table.

Table 14. Case Study 2 metric values.

\begin{tabular}{|c|c|c|c|c|}
\hline Metric Name & $\begin{array}{l}\text { Metric } \\
\text { Value }\end{array}$ & $\begin{array}{l}\text { Ideal } \\
\text { Metric } \\
\text { Value } \\
\end{array}$ & $\begin{array}{l}\text { Suggested } \\
\text { Target } \\
\text { Value } \\
\end{array}$ & Comment \\
\hline Rogue Change Days & unknown & 0 & 0 & Mechanisms are needed to detect rogue changes \\
\hline Password Crack Time & 0 days & $\infty$ & $30+$ days & $\begin{array}{l}\text { Use longer passwords and avoid LAN manager hash } \\
\text { of the password from being stored on the Windows } \\
\text { machines (see } \\
\text { http://support.microsoft.com/kb/299656). }\end{array}$ \\
\hline $\begin{array}{l}\text { Data Transmission } \\
\text { Exposure (Internet) }\end{array}$ & 0 & 0 & 0 & \\
\hline $\begin{array}{l}\text { Data Transmission } \\
\text { Exposure (Intranet) }\end{array}$ & 28 & 0 & 0 & $\begin{array}{l}\text { Avoid using unencrypted protocols through the } \\
\text { firewall. }\end{array}$ \\
\hline $\begin{array}{l}\text { 1. Reachability Count } \\
\text { (external) }\left(\mathrm{N}_{\mathrm{T}}\right)^{\mathrm{a}}\end{array}$ & 0 & 0 & 0 & External reachability count total \\
\hline 1. Physical $\left(\mathrm{N}_{\mathrm{p}}\right)$ & 0 & 0 & 0 & No physical access outside the control room \\
\hline 1. Services $\left(\mathrm{N}_{\mathrm{S}}\right)$ & 0 & 0 & 0 & External connectivity only through a VPN \\
\hline 1. Outgoing $\left(\mathrm{N}_{\mathrm{o}}\right)$ & 0 & 0 & 0 & firewall prevents outgoing connections \\
\hline $\begin{array}{l}\text { 2. Reachability Count } \\
(\text { local })\left(\mathrm{N}_{\mathrm{T}}\right)^{\mathrm{a}}\end{array}$ & 91 & 0 & 1 & $\begin{array}{l}\text { Local Reachability count total. From within control } \\
\text { room and/or from VPN }\end{array}$ \\
\hline 2. Physical $\left(\mathrm{N}_{\mathrm{p}}\right)$ & 4 & 0 & 0 & $\begin{array}{l}\text { HMI and EWS have unrestricted physical access USB } \\
\text { ports inside the control room. }\end{array}$ \\
\hline 2. Services $\left(\mathrm{N}_{\mathrm{S}}\right)$ & 87 & 0 & 1 & Suggest further firewall rules to restrict VPN access. \\
\hline 2. Outgoing $\left(\mathrm{N}_{\mathrm{o}}\right)$ & 0 & 0 & 0 & firewall prevents outgoing connections \\
\hline Worst Case Loss & unknown & $\$ 0$ & unknown & $\begin{array}{l}\text { Worst case power outage from a cyber attack is } \\
\text { estimated to be } 6 \text { hours duration. The dollar value of } \\
\text { such an outage has not been estimated. }\end{array}$ \\
\hline $\begin{array}{l}\text { Detection Mechanism } \\
\text { Deficiency Count }\end{array}$ & 46 & 0 & 0 & $\begin{array}{l}\text { There are no detection mechanisms on the system. } \\
\text { The addition of AV software and firewall restrictions } \\
\text { can reduce metric value to zero. }\end{array}$ \\
\hline $\begin{array}{l}\text { Vulnerability } \\
\text { Exposure (high } \\
\text { priority) }\end{array}$ & $\begin{array}{l}36,318 \\
\text { vuln. days }\end{array}$ & 0 & 0 & $\begin{array}{l}\text { Recommend the operating system be patched to } \\
\text { current level. The PLC vulnerability has no available } \\
\text { fix but PLC reachability improvement could mitigate } \\
\text { the vulnerability. }\end{array}$ \\
\hline $\begin{array}{l}\text { Vulnerability } \\
\text { Exposure } \\
\text { (low priority) }^{\text {b }}\end{array}$ & $\begin{array}{l}18,624 \\
\text { vuln. days }\end{array}$ & 0 & 0 & $\begin{array}{l}\text { All known vulnerabilities should be mitigated if } \\
\text { feasible. }\end{array}$ \\
\hline Attack Path Depth & 1 & $\infty$ & 2 & $\begin{array}{l}\text { Suggest further firewall rules to restrict VPN access } \\
\text { to increase attack path depth. }\end{array}$ \\
\hline Restoration Time & 72 hours & 0 & 5 hours & $\begin{array}{l}\text { Worst case restoration time is high because it requires } \\
\text { a complete software rebuild. }\end{array}$ \\
\hline
\end{tabular}




\section{SURVEY OF PREVIOUSLY PROPOSED TECHNICAL METRICS}

Thirty guides and standards documents (including, for example, references 10,11,12, and 13) were reviewed in search of technical metrics that have previously been defined and recommended. ${ }^{14}$ A sampling of security metrics used by some industries was also included in the investigation. Most of the metrics found in the standards and guides are not technical metrics. Only a few of the reviewed standards documents recommended the use of a specific metric or set of metrics. The metrics described in standards documents are generally provided as examples rather than as recommended metrics.

Survey results indicated the need for:

- A framework in which to think about control system security

- A set of practical technical metrics for use in better understanding security risks

Each of the identified technical metrics was analyzed by considering the circumstances in which the metric provides a meaningful security representation and relationship to risk. After evaluating the strengths and weaknesses of the various metrics it was determined that existing metrics have serious weaknesses. For example, many of the metrics were simply a percent of the system components that implemented a certain type of security control mechanism. However, the fractional implementation of a given security mechanism does not necessarily correlate to risk.

A specific metric defined in industry is "Average number of vulnerabilities per system component" This metric is easy to understand and it is easy to obtain estimates using automatic scanning tools. The problem with using an average is that all vulnerabilities and all components of the network are given equal weight. Consider the case where there is one easily exploitable vulnerability that allows penetration of a critical system component while there are zero known vulnerabilities on the other system components. Now consider a case where there are no known vulnerabilities on critical components and no vulnerabilities that allow penetration from an external site, but there are many minor vulnerabilities on noncritical system components. The former case is a high-risk situation, but the metric indicates low risk while the latter case is a low-risk situation, but the metric indicates high risk. The assumption that all vulnerabilities and all components are of equal value is false for most systems. The metric can be improved by counting the number of vulnerabilities for each group of components with similar security implications and for vulnerabilities with similar effects (e.g., external penetration versus privilege escalation).

The survey of existing technical metrics clearly demonstrated the need for a framework in which to think about control system security and the definition of a small set of practical technical metrics that you can use to gain better insight into your security risk. 


\section{SUMMARY}

The security framework for control systems consists of seven security dimensions, each related to risk. Based on case studies and reviews of control system security assessments, there is strong evidence that the framework's security dimensions capture many of the system attributes which correlate with a control system's risk exposure. As a result, the seven security dimensions are the recommended foundation for thinking about the security of your system and are the foundation for the ten recommended technical metrics.

The ten recommended technical security metrics support you in assessing whether your security risk exposure is increasing or decreasing over time. The recommended metrics have been applied to control systems and proven to be practical and useful. However, every system and facility is unique so there may be a need to select metrics or measurement technologies in line with your particular circumstances. That being the case, it is still important to have at least one metric for each of the seven security dimensions.

An important use of the recommended technical metrics is in tracking the improvement or degradation of your systems security posture along all seven dimensions of security. As the security posture improves, the risk to your control system from a cyber attack diminishes. Diligent use of the security framework and application of the technical metrics will aid you in making more effective security decisions for your control systems. 


\section{REFERENCES}

1. A. Jacquith, Security Metrics, Addison Wesley, 2007.

2. Tripwire, http://www.tripwire.com/products/index.cfm

3. Nessus, vulnerability scanner: http://www.nessus.org.

4. Metasploit http://www.metasploit.com

5. M. Schiffman, A Complete Guide to the Common Vulnerability Scoring System (CVSS), https://www.first.org/cvss/cvss-guide.html, Technical Report, Forum for Incident Response and Security Teams (FIRST), published June 7, 2005, Web site visited July 11, 2006.

6. Pwdump, http://www.darknet.org.uk/2006/10/download-pwdump-142-and-fgdump-134-windowspassword-dumping.

7. LC5, http://www.securityfocus.com/tools/1005.

8. John the Ripper, (http://www.openwall.com).

9. W. F. Boyer, M. A. McQueen, S. M. McBride, Control System Technical Security Metrics Report, INL Report to the Department of Homeland Security, INL/EXT-07-13562, December 2007.

10. Chemical Sector Cyber Security Program (CSCSP), Guidance for Addressing Cyber Security in the Chemical Industry, Technical Report, CSCSP, May 2006.

11. E. Chew, A. Clay, J. Hash, N. Bartol, A. Brown, Guide for Developing Performance Metrics for Information Security, NIST Special Publication 800-80, May 2006.

12. R. Ross, S. Katzke, A. Johnson, M. Swanson, and G. Rogers, "System Questionnaire with NIST SP 800-53: Recommended Security Controls for Federal Information Systems," Technical Report, NIST, References and Associated Security Control Mappings, Gaithersburg, Maryland, March 2006

13. M. Swanson, N. Bartol, J. Sabato, J. Hash, and L. Graffo, Security Metrics Guide for Information Technology Systems, NIST Special Publication 800-55, National Institute of Standards and Technology (NIST), Gaithersburg, Maryland, July 2003

14. INL Report to the Department of Homeland Security, Cyber Security Metrics, INL/EXT-06-12016, December 2006. 